- Case Report

\title{
Confusion, Faciobrachial Dystonic Seizures, and Critical Hyponatremia in a Patient with Voltage-Gated Potassium Channel Encephalitis
}

\author{
Julian Yaxley* \\ Department of Internal Medicine, Redcliffe Hospital, Redcliffe, Queensland, Australia
}

Autoimmune limbic encephalitis is a rare cause of encephalitic disease. It is associated with various target antigens and is difficult to diagnose, and experience with its treatment is limited. This case report describes a 69-year-old man, who presented with life-threatening hyponatremia and confusion, following several months of gradually worsening faciobrachial dystonic seizures. Faciobrachial dystonic seizures are a well-described feature classically observed in voltage-gated potassium channel autoimmune encephalitis. The presence of chronic hyponatremia without cognitive dysfunction, eventually culminating in an acute episode of encephalopathy and severe hyponatremia, is a pattern of natural history not previously documented in this condition.

Keywords: Seizure; Dystonia; Encephalitis; Hyponatremia 


\section{INTRODUCTION}

Autoimmune encephalitis is a rare disease which is only recently described. Its natural history is not fully understood although various responsible antigens have been identified. This case report describes a pathognomonic clinical sign which ultimately secured a diagnosis of VGKC limbic encephalitis and expedited appropriate treatment.

\section{CASE REPORT}

A 69-year-old man presented to his primary care physician with a recent onset of intermittent painful spasms of his right arm and face. Routine electrolytes were measured and were notable only for a serum sodium level of $130 \mathrm{mmol} / \mathrm{L}$ (reference range, 135 to $145 \mathrm{mmol} / \mathrm{L}$ ). The patient was diagnosed with cramps and advised to increase dietary salt intake. His spasms increased in frequency over the following months, but the patient did not seek further medical attention. Two months after his initial consultation with the general practitioner, the patient was transported to the emergency department by an ambulance with acute confusion and imbalance. This was accompanied by a near-constant, right-sided, spasmodic jerking. The patient's wife reported that until the day of presentation to the emergency department, his cognition and gait had been completely normal. During the twitching episodes, his level of consciousness had never been impaired.

The patient's past medical history was notable for an ischemic stroke 8 years earlier, with mild residual weakness of the upper left limb, hypertension, and post-traumatic stress disorder. His medications included aspirin, atorvastatin, irbesartan, and venlafaxine. The patient was a retired soldier and a non-smoker, who consumed alcohol only socially.

On examination in the emergency department, the patient was confused and agitated. He reacted to voice by opening his eyes, but he could only manage unintelligible speech. He was uncooperative and detailed neurological examination was difficult. All limbs appeared to move equally, and pupils were isocoric and sluggishly reactive to light. Brief stereotyped involuntary contractions of the right side of the face and upper limb were noted every 2-3 minutes. These were characterized by facial contortion, and right elbow and wrist flexion, and finger extension. There were no features of infection or trauma.

The results of serum biochemistry on arrival were notable for an abnormal sodium concentration of $113 \mathrm{mmol} / \mathrm{L}$ and serum osmolality of $241 \mathrm{mmol} / \mathrm{L}$ (range, 275 to $295 \mathrm{mmol} / \mathrm{L}$ ). A baseline serum sodium obtained by the family physician 12 months earlier was within the normal range at $136 \mathrm{mmol} / \mathrm{L}$. Urine sodium concentration was 122 $\mathrm{mmol} / \mathrm{L}$ with osmolality of $541 \mathrm{mmol} / \mathrm{L}$. A full blood count and tests for inflammatory markers returned results within the normal ranges. Emergency brain computed tomography did not reveal any notable changes.

The patient's mental status improved rapidly, following urgent treatment with $3 \%$ hypertonic saline and, subsequently, fluid restriction. Electroencephalogram (EEG) showed frontotemporal slowing with no epileptiform activity. Magnetic resonance imaging (MRI) of the brain demonstrated only deep white matter hyperintensities, consistent with chronic small vessel ischemia. Analysis of cerebrospinal fluid (CSF), obtained by lumbar puncture, did not detect abnormalities in protein or glucose levels, microscopic examination, or bacterial culture results.

Twitches remained obvious and frequent over the following week. These were thought to be consistent with faciobrachial dystonic seizures (FDSs), typical of one variant of autoimmune limbic encephalitis related to voltage-gated potassium channel antibodies (VGKC antibodies). Empiric treatment with intravenous methylprednisolone and immunoglobulin was commenced. A full resolution of the patient's abnormal movements was observed within several days.

Serum paraneoplastic antibodies were negative. Reanalysis of the CSF identified elevated VGKC antibodies ( 589 pM, normal range $<85$ $\mathrm{pM})$, leading to a final diagnosis of voltage-gated potassium channel encephalitis. This was thought to have caused the syndrome of inappropriate secretion of anti-diuretic hormone (SIADH), which induced critical hypoosmolar hyponatremia, with confusion and ataxia.

Following hospitalization, the patient remained stable in the community on a regimen of daily oral prednisone and monthly intravenous immunoglobulin (IVIG).

\section{DISCUSSION}

Patients with encephalitic syndromes present a diagnostic challenge. Autoimmune limbic encephalitis is a recently described entity, whose pathogenesis remains poorly understood. The inflammatory process is confined largely to structures of the limbic system and several antigenic targets have been implicated including VGKCs, the glutamate NMDA (N-methyl-D-aspartate) and AMPA ( $\alpha$-amino-3-hydroxy5-methylisoxazole-4-propionic acid) receptors, and GABA (gammaaminobutyric acid) receptors.

VGKC encephalitis is a rare disease and an uncommon variety of limbic encephalitis. The true incidence of VGKC encephalitis is unknown, although it is increasingly recognized. While this condition is conventionally attributed to an autoimmune reaction against the VGKC, it is now known that antibodies used in laboratories to test for VGKC in fact act on leucine-rich glioma-inactivated protein 1 (LGI1), a neuronal secreted protein, which is a structural component of the VGKC complex. ${ }^{1,2)}$ Some authors, therefore, refer to anti-LGIl antibody or anti-VGKC-complex antibodies instead of anti-VGKC antibody, and may replace the term 'VGKC encephalitis' with 'LGI1 encephalitis.')

Many cases of autoimmune limbic encephalitis are paraneoplastic. In the VGKC subtype the incidence of cancer is relatively lower at $11 \% .{ }^{3)}$ Features of VGKC encephalitis typically precede detection of malignancy. ${ }^{3)}$ The tumors most commonly associated with VGKC encephalitis are small cell lung cancer and thymoma.

Reported clinical manifestations of VGKC encephalitis include altered memory and behavior, confusion, drowsiness, and psychosis. The acute delirium observed in our case, with previously normal men- 
tal function, is an unusual presentation of the disease. Typically, almost all patients demonstrate subtle cognitive dysfunction for at least 1 week prior to the development of a clinically obvious syndrome. ${ }^{4)}$ Dystonia and chorea are frequently seen, and are often the first sign of VGKC encephalitis, predating other symptoms by weeks, as was demonstrated in our case. FDSs are a pathognomonic feature of VGKC encephalitis. ${ }^{5,6)}$ These are not true seizures, but rather dystonic reactions that are not observed in other forms of autoimmune limbic encephalitis. FDSs usually evolve over weeks or months, but fulminant presentations do occur.

Establishing the diagnosis of VGKC encephalitis can be difficult. There is no single diagnostic test, although lumbar puncture and CSF analysis are central. Most patients have raised CSF protein and many have a lymphocytic pleocytosis. ${ }^{3)}$ VGKC antibodies are detected in the CSF in the great majority of patients. ${ }^{3,7)}$ The VGKC antibodies may also be identified in the serum, although false positives are more frequent. The EEG is usually non-specific, with frontotemporal or bitemporal generalized slowing. Some patients have epileptiform activity. Neuroimaging can support the diagnosis, but findings are not conclusive. Brain MRI results usually find high-signal changes in medial temporal lobes, which are enhanced on T2-weighted scans. ${ }^{3)}$ Fludeoxyglucose positron emission tomography (FDG-PET) results often reveal hypermetabolism in the basal ganglia and the temporal lobes, and, at the later stages, the disease may demonstrate hypometabolism in the frontotemporal cortex. ${ }^{8,9)}$ Clinicians should be aware that, because VGKC encephalitis sometimes represents a paraneoplastic syndrome, evaluation for occult malignancy should be considered for every patient.

Approximately $80 \%$ of cases of VGKC encephalitis develop hyponatremia at some time during the illness, generally as a result of SIADH. ${ }^{10)}$ The pattern of hyponatremia observed in our case is unique. Previously, low serum sodium has only been reported in patients with prior evidence of encephalopathy, such as subacute memory impairment or worsening irritability. ${ }^{10)}$ Asymptomatic, mild hyponatremia was apparent in our patient 2 months prior to the sudden onset of confusion, and, by the time of presentation, had progressed to critically low serum sodium concentration. Given the prompt improvement in his mental state, upon reversal of hyponatremia, it is hypothesized that this patient's encephalopathy was a consequence of critical hyponatremia, rather than cognitive deficits from encephalitis per se.

Most patients experience full recovery with immunomodulatory therapy, and relapse is uncommon. Some exhibit subtle residual neurological deficits. Earlier detection is presumed to lead to better neurologic outcomes. ${ }^{3,10)}$ There is very little scientific evidence to direct treatment for autoimmune encephalitis; with empirical experience, corticosteroids and IVIG have become the most commonly used agents. ${ }^{10)}$ Other immunosuppressive treatments that have been trialed successfully, include plasma exchange, rituximab, and mycophenolate. Anticonvulsants have little impact on faciobrachial dystonic seizures, but are indicated in true seizures. ${ }^{5)}$

In summary, VGKC encephalitis is a rare disease, which, if detected, is amenable to treatment. The pattern of presentation in our case has not been previously reported in medical literature. While hyponatremia is a common finding in VGKC encephalitis, asymptomatic, chronically low serum sodium preceding an acute delirium is unusual. The combination of FDSs and mild hyponatremia thereby provided an early opportunity for diagnosis, which was missed and ultimately led to an acute life-threatening process. The fact that this patient presented initially to primary care highlights the importance of the understanding of VGKC encephalitis by general practitioners.

\section{CONFLICT OF INTEREST}

No potential conflict of interest relevant to this article was reported.

\section{REFERENCES}

1. Lai M, Huijbers MG, Lancaster E, Graus F, Bataller L, Balice-Gordon R, et al. Investigation of LGIl as the antigen in limbic encephalitis previously attributed to potassium channels: a case series. Lancet Neurol 2010;9:776-85.

2. Tofaris GK, Irani SR, Cheeran BJ, Baker IW, Cader ZM, Vincent A. Immunotherapy-responsive chorea as the presenting feature of LGI1-antibody encephalitis. Neurology 2012;79:195-6.

3. Wingfield T, McHugh C, Vas A, Richardson A, Wilkins E, Bonington A, et al. Autoimmune encephalitis: a case series and comprehensive review of the literature. QJM 2011;104:921-31.

4. Vincent A, Buckley C, Schott JM, Baker I, Dewar BK, Detert N, et al. Potassium channel antibody-associated encephalopathy: a potentially immunotherapy-responsive form of limbic encephalitis. Brain 2004;127(Pt 3):701-12.

5. Sen A, Wang J, Laue-Gizzi H, Lee T, Ghougassian D, Somerville ER. Pathognomonic seizures in limbic encephalitis associated with antiLGI1 antibodies. Lancet 2014;383:2018.

6. Chang BS. The face (and arm) of treatment for seizures in VGKC/LGI1 antibody-associated limbic encephalitis. Epilepsy Curr 2014;14:180-2.

7. Lawn ND, Westmoreland BF, Kiely MJ, Lennon VA, Vernino S. Clinical, magnetic resonance imaging, and electroencephalographic findings in paraneoplastic limbic encephalitis. Mayo Clin Proc 2003;78:1363-8.

8. Navarro V, Kas A, Apartis E, Chami L, Rogemond V, Levy P, et al. Motor cortex and hippocampus are the two main cortical targets in LGI1-antibody encephalitis. Brain 2016;139(Pt 4):1079-93.

9. Basu S, Alavi A. Role of FDG-PET in the clinical management of paraneoplastic neurological syndrome: detection of the underlying malignancy and the brain PET-MRI correlates. Mol Imaging Biol 2008;10:131-7.

10. Irani SR, Michell AW, Lang B, Pettingill P, Waters P, Johnson MR, et al. Faciobrachial dystonic seizures precede Lgil antibody limbic encephalitis. Ann Neurol 2011;69:892-900. 